\title{
Traitement médiatique des MOOC en tant qu'innovation numérique en éducation : analyse du positionnement des chercheurs, des administrations universitaires et des directions des plateformes de MOOC
}

\author{
Media treatment of MOOCs as digital innovation in education: \\ analysis of the positioning of researchers, university administrations \\ and MOOC administrators

\section{Tratamiento mediático de los MOOC como innovación digital en educación: análisis del posicionamiento de investigadores, administraciones universitarias y direcciones de plataformas MOOC}

Simon Collin, Hamid Saffari et Demba Seck

Volume 21, numéro 3, 2019

Une approche sociocritique : quels apports à l'étude du numérique en éducation?

URI : https://id.erudit.org/iderudit/1067711ar

DOI : https://doi.org/10.7202/1067711ar

Aller au sommaire du numéro

Éditeur(s)

Faculté d'éducation, Université de Sherbrooke

ISSN

1911-8805 (numérique)

Découvrir la revue

Citer cet article

Collin, S., Saffari, H. \& Seck, D. (2019). Traitement médiatique des MOOC en tant qu'innovation numérique en éducation : analyse du positionnement des chercheurs, des administrations universitaires et des directions des plateformes de MOOC. Nouveaux cahiers de la recherche en éducation, 21(3), 116-134. https://doi.org/10.7202/1067711ar

\section{Résumé de l'article}

Entre 2012 et 2014, les MOOC (Massive Open Online Courses), en tant qu'innovation numérique en pédagogie universitaire, ont fait l'objet d'un fort traitement médiatique. Loin d'être une simple courroie de transmission, ce traitement médiatique peut être compris comme une mise en scène de différents acteurs (politiques, administratifs, scientifiques, éducatifs) articulés autour d'un imaginaire des MOOC en tant qu'innovation. Certains aspects de cet imaginaire ont déjà pu être identifiés lors d'une précédente étude (Collin et Saffari, 2015), ainsi que trois types d'intervenants principalement cités ou interviewés par les médias au regard des MOOC : les administrations universitaires, les directions des plateformes de MOOC et les chercheurs étudiant les technologies en éducation. En revanche, la manière dont le traitement médiatique des MOOC a mis en scène ces trois types d'acteurs n'a pas fait l'objet d'analyse. C'est ce que nous nous proposons d'approfondir dans le cadre du présent article, dont l'objectif est de mieux comprendre comment le traitement médiatique des MOOC positionne les chercheurs, les administrations universitaires et les directions de plateformes MOOC au sein de l'imaginaire qu'ils véhiculent. Pour ce faire, nous avons mené une analyse secondaire du même corpus d'articles journalistiques qui a servi à la première étude (voir Collin et Saffari, 2015), en nous focalisant cette fois-ci sur les différents intervenants cités ou interviewés dans les articles journalistiques (p. ex., chercheurs, directions des plateformes MOOC, administrations universitaires). Les résultats de l'analyse de contenu permettent d'identifier, pour chaque type d'intervenant, des positionnements complémentaires au sein du traitement médiatique des MOOC. 


\title{
Traitement médiatique des MOOC en tant qu'innovation numérique en éducation: analyse du positionnement des chercheurs, des administrations universitaires et des directions des plateformes de MOOC
}

\section{Simon Collin}

\author{
Université du Québec à Montréal
}

Hamid Saffari

Université du Québec en Outaouais

Demba Seck

\section{Université du Québec à Montréal}

\begin{abstract}
Résumé
Entre 2012 et 2014, les MOOC (Massive Open Online Courses), en tant qu'innovation numérique en pédagogie universitaire, ont fait l'objet d'un fort traitement médiatique. Loin d'être une simple courroie de transmission, ce traitement médiatique peut être compris comme une mise en scène de différents acteurs (politiques, administratifs, scientifiques, éducatifs) articulés autour d'un imaginaire des MOOC en tant qu'innovation. Certains aspects de cet imaginaire ont déjà pu être identifiés lors d'une précédente étude (Collin et Saffari, 2015), ainsi que trois types d'intervenants principalement cités ou interviewés par les médias au regard des MOOC: les administrations universitaires, les directions des plateformes de MOOC et les chercheurs étudiant les technologies en éducation. En revanche, la manière dont le traitement médiatique des MOOC a mis en scène ces trois types d'acteurs n'a pas fait l'objet d'analyse. C'est ce que nous nous proposons d'approfondir dans le cadre du présent article, dont l'objectif est de mieux comprendre comment le traitement médiatique des MOOC positionne les chercheurs, les administrations universitaires et les directions de plateformes MOOC au sein de l'imaginaire qu'ils véhiculent. Pour ce faire, nous avons mené une analyse secondaire du même corpus d'articles journalistiques qui a servi à la première étude (voir Collin et Saffari, 2015), en nous focalisant cette fois-ci sur les différents intervenants cités ou interviewés dans les articles journalistiques (p. ex., chercheurs, directions des plateformes MOOC, administrations universitaires). Les résultats de l'analyse de contenu permettent d'identifier, pour chaque type d'intervenant, des positionnements complémentaires au sein du traitement médiatique des MOOC.
\end{abstract}

Mots clés: MOOC, traitement médiatique, positionnement des acteurs de l'innovation 


\section{Media treatment of MOOCs as digital innovation in education: analysis of the positioning of researchers, university administrations and MOOC administrators}

\section{Abstract}

Between 2012 and 2014, Massive Open Online Courses (MOOCs) received considerable media coverage as a digital innovation in university pedagogy. Far from being a mere conveyor belt of information, this media treatment can be understood as a staging of different actors (political, administrative, scientific and educational) around an imaginary of the MOOC as an innovation. Some aspects of this imaginary have already been identified in a previous study (Collin \& Saffari, 2015), along with three types of stakeholders typically quoted or interviewed by the media with regard to MOOCs: university administrations, MOOC administrators and researchers studying technologies in education. However, the way the media treatment of MOOCs has staged these three types of actors has not been analyzed. This article sets out to address just this subject, with the aim of better understanding how the media treatment of MOOC positions researchers, university administrations and MOOC administrators within the imaginary that they convey. To this end, we conducted a secondary analysis of the same body of journalistic articles used for a first study (see Collin \& Saffari, 2015), focusing this time on the various stakeholders quoted or interviewed in the articles (e.g., researchers, MOOC administrators, university administrations). For each type of stakeholder, the results of the content analysis help identify complementary positions within the media treatment of MOOCs.

Keywords: MOOC, media treatment, positioning of innovation actors

\section{Tratamiento mediático de los MOOC como innovación digital en educación: análisis del posicionamiento de investigadores, administraciones universitarias y direcciones de plataformas MOOC}

\section{Resumen}

Entre 2012 y 2014, los cursos masivos abiertos en línea (MOOC), recibieron mucha atención de los medios, como innovación digital en la pedagogía universitaria. Lejos de ser un simple espacio de transmisión, este tratamiento mediático puede entenderse como una puesta en escena de diferentes actores (políticos, administrativos, científicos, educativos) articulados en torno al imaginario de los MOOC como una innovación. Algunos aspectos de este imaginario han sido identificados en un estudio previo (Collin y Saffari, 2015), así como tres tipos de partes interesadas principalmente citados o entrevistados por los medios de comunicación con respecto a los MOOC: las administraciones universitarias, las direcciones de plataformas MOOC y los investigadores que estudian las tecnologías en educación. Sin embargo, no se ha analizado la forma en que el tratamiento mediático de los MOOC ha organizado estos tres tipos de actores. Esto es lo que pretendemos profundizar en este artículo, cuyo objetivo es comprender mejor cómo el tratamiento mediático del MOOC posiciona a los investigadores, las administraciones universitarias y los administradores de la plataforma MOOC a partir del imaginario que estos manejan. Para hacer esto, realizamos un análisis secundario del mismo cuerpo de artículos periodísticos que se utilizó para el primer estudio (ver Collin y Saffari, 2015), centrándose esta vez en las diversas partes interesadas citadas o entrevistadas en lo artículos periodísticos (por ejemplo, investigadores, administradores de la plataforma MOOC, administraciones universitarias).Los resultados del análisis de contenido permiten identificar, para cada tipo de participante, posiciones complementarias del procesamiento mediático de los MOOC.

Palabras clave: $\quad$ MOOC, tratamiento mediático, posicionamiento de actores innovadores 


\section{Contexte}

\subsection{Le traitement médiatique des MOOC en tant qu'innovation numérique en éducation}

Comme toute innovation numérique en éducation, il est possible d'inscrire les MOOC (Massive Open Online Courses), tels qu'on les connaît aujourd'hui, dans une filiation historique dont ils sont tributaires. Mentionnons, par exemple, les liens entre la formation par correspondance et la formation à distance (Noble, 2002), et plus récemment les MOOC. Ces derniers ont d'ailleurs existé avant même qu'on les nomme ainsi. En effet, certains auteurs (voir Karsenti, 2013) retracent l'origine des MOOC à l'initiative irlandaise Advance Learning Interactive Systems Online ${ }^{1}$ qui a commencé en 2007 et dont le but était de développer les compétences professionnelles en lien avec le marché du travail. Le terme "MOOC" serait toutefois attribué à Cormier et Alexander pour désigner le cours Connectivism and Connective Knowledge qu'a dispensé Downes en 2008 (Karsenti, 2013).

Depuis les années 1960, les médias sont partie prenante des discours et de la diffusion des innovations numériques en éducation (Forestier, 2014). Évoquant le cas de l'Angleterre, Wilby (2014) souligne que pratiquement tous les journaux britanniques nationaux disposent aujourd'hui d'un correspondant en charge de l'éducation, couvrant l'actualité des écoles et des établissements d'enseignement supérieur, notamment en ce qui a trait aux innovations numériques. Les MOOC n'ont pas fait exception. Leur traitement médiatique a débuté en 2011 avec le cours Introduction to Artificial Intelligence dispensé par Thrun et Norvig et s'est emballé dans les années 2012 et 2013, avec l'apparition d'un certain nombre de plateformes de MOOC, tels que Coursera ${ }^{2}$, EdX $^{3}$, Eliademy ${ }^{4}$, FutureLearn ${ }^{5}$, Open2Study ${ }^{6}$ ou encore Udacity ${ }^{7}$ de sorte qu'il est possible de penser que les MOOC ont été largement médiatisés. Il en a résulté une éruption de nouveaux MOOC.

\subsection{Traitement médiatique et imaginaire de l'innovation numérique en éducation}

Comme pour toute innovation numérique en éducation (et au-delà), le traitement médiatique des MOOC constitue une part importante de leur diffusion dans le corps social et éducatif. Pour Garçon (2006), «il n'est de techniques sans capacité d'inventer. Et il n'est de capacité d'inventer sans imaginaire. Dans son lien à la technique, la première fonction de l'imaginaire est de faciliter l'invention; la seconde est de faciliter l'acceptation

\footnotetext{
Alison. Repéré à https://www.alison.com.

Coursera. Repéré à https://www.coursera.org.

edX. Repéré à https://www.edx.org.

Eliademy. Repéré à https://www.eliademy.com [hors ligne].

Future Learn. Repéré à https://www.futurelearn.com.

Open2Study. Repéré à https://www.open2study.com.

Udacity. Repéré à https://www.udacity.com.
} 
de cette invention" (p. 224). Pour souligner ce lien indissociable entre innovation numérique et imaginaire, Balandier parle de "techno-imaginaire" (Chanial, 1994). Dans cette perspective, l'innovation numérique est duale: fonctionnelle et fictionnelle. Elle ne peut pas être dissociée des fictions discursives qui l'accompagnent, la socialisent et la naturalisent. Ces dernières mobilisent un imaginaire qui peut en appeler autant à la fascination qu'à la peur. Ainsi, Le Blanc (2017) remarque que, pour le cas de la France, c'est "un discours, glorieux et finalement intemporel, qui jalonne les grandes étapes qui ont marqué, en trente ans, la dizaine de plans numériques pour l'école» (p. 62).

Pour le cas des MOOC, l'imaginaire prend notamment la forme d'une transformation de l'enseignement supérieur à moindre prix, comme l'expriment Oliveri et Rasse (2017): "L'argument économique est un axe de communication privilégié pour valoriser les MOOC auprès des collectivités soucieuses de réduire leurs dépenses, mais aussi auprès d'un public en attente de solutions techniciennes gratuites ou à faible coût. En l'état, l'idée selon laquelle les MOOC se multiplieraient inexorablement et représenteraient de manière quasi inéluctable le futur de l'enseignement est diffusée dans l'opinion publique, via une sphère médiatique bienveillante» (p. 114). On retrouve ici un discours technodéterministe qu'une approche sociocritique (Collin, Guichon, Ntebutse, 2015) permet précisément d'identifier et de déconstruire.

\subsection{Traitement médiatique et mise en scène des acteurs}

Comme le soulignent Gérard et Proteau (2005), le traitement médiatique d'une innovation numérique implique une mise en scène des relations complexes qu'entretiennent les acteurs politiques, administratifs, scientifiques et éducatifs au sein de l'imaginaire technique. Ce faisant, il contribue à façonner l'imaginaire de l'innovation, mais aussi de ses divers acteurs (Millerand, 1999).

Le traitement médiatique de l'innovation numérique n'est donc pas neutre pour plusieurs raisons. D'une part, il contribue à valoriser les points de vue et les intérêts de certains acteurs plutôt que d'autres, notamment ceux qui sont en position de force, et offrent donc un traitement partiel et partial des acteurs de l'innovation (Gérard et Proteau, 2005). D'autre part, l'imaginaire qu'il véhicule «est bien différent de celui des inventeurs, et il ne correspond pas à celui des publics. [...]. Leur parole complexifie la relation dialectique entre technique et imaginaire" (Garçon, 2006, p. 227). Ainsi, le traitement médiatique des innovations numériques en éducation, loin d'être une simple courroie de transmission, peut être compris comme une mise en scène de différents acteurs (politiques, administratifs, scientifiques, éducatifs) articulés autour d'un imaginaire de l'innovation.

Pour le cas des MOOC, une première étude que nous avons menée (Collin et Saffari, 2015) nous a permis de relever certains aspects de l'imaginaire que les médias consultés 
ont véhiculés à leur sujet. Entre autres, les MOOC étaient associés à la démocratisation de l'accès à l'enseignement supérieur, à une visibilité académique et culturelle accrue des universités et au changement de la pédagogie universitaire. En outre, nous avons pu à cette occasion identifier trois principaux types d'intervenants qui étaient cités ou interviewés régulièrement par les médias au regard des MOOC: les administrations universitaires et les directions des plateformes de MOOC, qui sont également cités par Roland et Uyttebrouck (2015), ainsi que les chercheurs étudiant les technologies en éducation, qui sont mobilisés pour leur expertise scientifique au regard des MOOC.

En revanche, la manière dont les médias ont mis en scène ces trois types d'acteurs n'a pas fait l'objet d'analyse, de sorte que la question reste ouverte de savoir comment le traitement médiatique des MOOC a articulé ses acteurs au sein de l'imaginaire des MOOC. C'est ce que nous nous proposons d'approfondir dans le cadre du présent article, dont l'objectif est de mieux comprendre comment le traitement médiatique des MOOC positionne les chercheurs, les administrations universitaires et les directions de plateformes de MOOC au sein de l'imaginaire qu'ils véhiculent. Plus largement, cette étude souhaite contribuer à mieux comprendre la manière dont les discours, par les imaginaires de l'innovation et la mise en scène des acteurs concernés, participent à l'intégration du numérique en éducation. Bien que les discours sur le numérique en éducation soient peu étudiés, ils constituent à notre sens une voie d'entrée très pertinente dans l'imaginaire qui leur est inhérent et qui est susceptible de jouer sur la disposition des différents acteurs concernés. En nous intéressant ainsi aux discours médiatiques, nous nous inscrivons dans la continuité de quelques travaux ayant abordé le cas des discours sur le numérique en éducation, notamment ceux de Moatti (2010) sur les discours politiques et administratifs officiels sur le numérique entre les années 1977 et 2009 en France.

\section{Méthodologie}

Nous avons mené une analyse secondaire sur le même corpus d'articles journalistiques généralistes ayant servi à la rédaction d'un précédent article (voir Collin et Saffari, 2015). Nous commençons par rappeler comment le corpus d'articles journalistiques généralistes a été constitué et avec quels codes et sous-codes il a été analysé. Nous expliquons ensuite comment nous avons procédé à l'analyse des types d'intervenants à partir de cette première analyse.

\subsection{Collecte de données}

Pour effectuer notre récolte de données, plusieurs mots-clés relatifs aux MOOC ont été appliqués aux sites web Eureka, Google actualités et Twitter. Parmi les critères d'inclusion, les articles journalistiques devaient obligatoirement 1 ) être rédigés soit en français, soit 
en anglais; 2) être publiés dans des journaux généralistes ayant une échelle nationale ou régionale (p. ex., The New York Times, Le Figaro, etc.); 3) aborder les MOOC d'un point de vue critique (article d'opinion) et non pas d'un point de vue descriptif (c.-à-d., qui décrit ce que sont les MOOC) ou informatif (c.-à-d., qui communiquent des informations de type factuel, comme le fait qu'une université a lancé un nouveau MOOC); 4) être publiés entre janvier 2012 et décembre 2014 (date de la collecte de données). Au final, 177 articles ont été sélectionnés dont 88 étaient en anglais et 89 en français.

\subsection{Analyse initiale des données}

L'analyse de contenu (van der Maren, 1996) était l'approche méthodologique adoptée pour analyser les articles journalistiques. Pour ce faire, nous avons eu recours au logiciel d'analyse QDA Miner (Karsenti, Komis, Depover et Collin, 2011). Cinq codes principaux, ainsi que des sous-codes associés, ont émergé de notre analyse de contenu. Le tableau 1 présente les définitions des cinq codes principaux alors que les sous-codes seront présentés au fur et à mesure des résultats.

Tableau 1 - Définition des codes principaux ayant émergé de l'analyse de contenu des articles journalistiques généralistes (adaptée de Collin et Saffari, 2015)

\begin{tabular}{|c|l|}
\hline $\begin{array}{c}\text { Nom des codes } \\
\text { principaux }\end{array}$ & \multicolumn{1}{c|}{ Définition } \\
\hline $\begin{array}{c}\text { Contextualisation } \\
\text { historique et sociale }\end{array}$ & $\begin{array}{l}\text { Ce code a été attribué aux propos qui inscrivent les MOOC dans un contexte } \\
\text { plus large, qu'il soit historique (continuité historique) ou social (environnement } \\
\text { social). } \\
\text { Par exemple, dire que les MOOC sont susceptibles de répondre au défi de la } \\
\text { démocratisation de l'accès à l'enseignement supérieur dans certains pays en } \\
\text { voie de développement relève de ce code, car il permet d'inscrire les MOOC } \\
\text { au sein de certains enjeux sociaux auxquels l'enseignement supérieur fait } \\
\text { face dans certaines sociétés. }\end{array}$ \\
\hline $\begin{array}{c}\text { Projection historique } \\
\text { et sociale }\end{array}$ & $\begin{array}{l}\text { Ce code a été attribué aux propos qui prédisent des changements et des } \\
\text { effets occasionnés par les MOOC. Dans une perspective critique, la projection } \\
\text { historique et sociale peut témoigner d'un discours idéologique (Friesen, } \\
\text { 2008) si elle n'est appuyée sur aucune contextualisation (voir premier code } \\
\text { principal), car il s'agit alors d'une projection «désincarnée" des réalités sociales } \\
\text { et historiques, qui ne bénéficie d'aucun cadre de référence pour l'orienter. } \\
\text { Autrement dit, la projection n'est pas idéologique en soi, mais elle peut le } \\
\text { devenir lorsqu'aucune contextualisation substantielle ne l'accompagne. } \\
\text { Par exemple, dire que les MoOC vont entraîner la fermeture des universités } \\
\text { sur campus relève de la projection. Elle peut s'avérer idéologique si cette } \\
\text { projection n'est pas étayée par une contextualisation sociale et historique } \\
\text { qui la rend plausible. }\end{array}$ \\
\hline $\begin{array}{c}\text { Constat d'enjeux } \\
\text { nouveaux }\end{array}$ & $\begin{array}{l}\text { Ce code a été attribué aux propos qui décrivent des aspects problématiques } \\
\text { des MOOC qui n'étaient pas anticipés initialement et pour lesquels il s'agit } \\
\text { de trouver des solutions. } \\
\text { Par exemple, dire que les Mooc nécessitent des techniques d'authentification } \\
\text { des étudiants pour certifier leurs évaluations, relève d'un enjeu qui est apparu } \\
\text { de plus en plus pressant au fur et à mesure que les universités faisaient } \\
\text { l'expérience des Mooc. }\end{array}$ \\
\hline
\end{tabular}




\begin{tabular}{|c|l|}
\hline Mise en doute critique & $\begin{array}{l}\text { Ce code a été attribué aux propos qui dénoncent des projections historiques } \\
\text { et sociales (voir deuxième code principal) lorsque ces dernières se sont } \\
\text { avérées infondées. } \\
\text { Par exemple, dire que les MOOC ne contribuent pas à la démocratisation } \\
\text { annoncée de l'accès à l'enseignement supérieur puisqu'ils attirent des } \\
\text { individus déjà diplômés est une mise en doute critique de la projection } \\
\text { historique selon laquelle les MOOC vont permettre de démocratiser l'accès à } \\
\text { l'enseignement supérieur. }\end{array}$ \\
\hline Perspectives futures & $\begin{array}{l}\text { Ce code a été attribué aux propos qui envisagent les perspectives d'avenir } \\
\text { des MOOC. } \\
\text { Par exemple, dire que les MOOC ont un certain potentiel pour la formation } \\
\text { en entreprise s'inscrit dans ce code. }\end{array}$ \\
\hline
\end{tabular}

\subsection{Analyse secondaire des données par type d'intervenant}

Pour procéder à l'analyse secondaire, nous avons effectué un codage du même corpus d'articles journalistiques généralistes (voir Collin et Saffari, 2015) en ciblant les types d'intervenants régulièrement cités ou interviewés, à savoir, les chercheurs, les administrations universitaires et les directions de plateformes de MOOC. Nous avons ensuite repris le codage initial du corpus (cing codes et les sous-codes associés) afin de caractériser les propos des intervenants. Comme la représentation des types d'intervenant est inégale dans le corpus analysé (voir section «Types d'intervenant interviewés ou cités») et dans les codes principaux retenus (voir section «Distribution des types d'intervenants selon les cinq codes principaux"), nous avons mis chacun d'eux en pourcentage absolu de façon à pouvoir les comparer. Pour la même raison, nous basons nos comparaisons non pas tant sur les pourcentages effectifs d'intervention de chaque type d'intervenant que sur les rangs qu'ils occupent les uns par rapport aux autres.

\section{Résultats}

Pour une première prise en main de nos résultats, nous commençons par présenter le profil des auteurs des articles qui ont été analysés ainsi que la proportion des trois types d'intervenants cités ou interviewés, à savoir, les chercheurs, les directions des plateformes de MOOC et les administrations universitaires. Nous exposons ensuite la distribution de ces trois types d'intervenants selon les codes principaux, puis selon les sous-codes de chacun d'eux.

\subsection{Profil des auteurs des articles}

Commençons par préciser qui sont les auteurs des articles analysés, car ils sont porteurs d'intérêts potentiellement distincts en fonction de leur statut. Pour ce faire, nous avons fait une recherche sur chacun d'entre eux pour vérifier leurs profils, soit sur le site de leurs journaux, soit en cherchant sur internet. À titre d'exemple, pour déterminer si un 
auteur est journaliste généraliste ou spécialisé en éducation, nous avons vérifié le sujet et le titre de la majorité de ses articles en ligne.

En grande majorité $(65 \%)$, les auteurs des articles étudiés sont des journalistes généralistes, ce qui est congruent avec les journaux (eux-mêmes généralistes) ciblés lors de la collecte de données. Les journalistes spécialisés en éducation viennent au second rang avec $12 \%$ des auteurs des articles analysés, suivis des journalistes spécialisés en technologies (6\%). Bien que de façon secondaire (16\%), il est intéressant de noter que les chercheurs apparaissent également comme auteurs de certains articles. Enfin, les journalistes spécialisés sur les TIC en éducation sont peu nombreux ( $2 \%)$, ce qui s'explique sans doute par le fait que leur expertise est rare.

Ainsi, les articles étudiés font intervenir une diversité d'auteurs, à la fois en ce qui a trait au statut (journalistes ou chercheurs) et à la spécialisation. Les chercheurs, plus précisément, ont contribué doublement à façonner les discours sur les MOOC: certains en tant qu'auteurs; d'autres en tant qu'intervenants interviewés ou cités, comme nous pouvons le voir maintenant.

\subsection{Types d'intervenant interviewé ou cité}

En nous intéressant aux types d'intervenants interviewés ou cités dans les articles étudiés, nous remarquons que les chercheurs sont de loin les plus sollicités pour discourir sur les MOOC, avec 87 articles (soit $53 \%$ ) référant à leurs propos. Les administrations universitaires (en particulier les recteurs de grandes universités américaines telles que Stanford, Harvard, etc.) et les directions de plateformes MOOC (principalement celles de Coursera, Edex et Udacity) forment les deuxième et troisième groupes cités, quoiqu'avec une représentation moitié moins grande que celle des chercheurs. Les discours journalistiques généralistes sur les MOOC semblent donc avoir largement fait usage de l'expertise scientifique.

Une analyse chronologique des trois types d'intervenants permet de noter qu'ils apparaissent à des moments distincts dans le traitement médiatique des MOOC (voir figure 1). Les plus sollicités durant l'année 2012, qui marque le début de l'attention médiatique sur les MOOC, sont les directions des plateformes de MOOC. L'année 2013 est dominée par les administrations universitaires. Ces deux types d'intervenants fléchissent toutefois dans la première moitié de l'année 2014 et laissent ainsi place aux chercheurs. Finalement, les trois types d'intervenants se maintiennent ou sont en baisse dans la deuxième moitié de l'année 2014, ce qui semble s'expliquer par un essoufflement des MOOC comme objet médiatique (Collin et Saffari, 2015). 
Figure 1 - Types d'intervenant interviewé ou cité

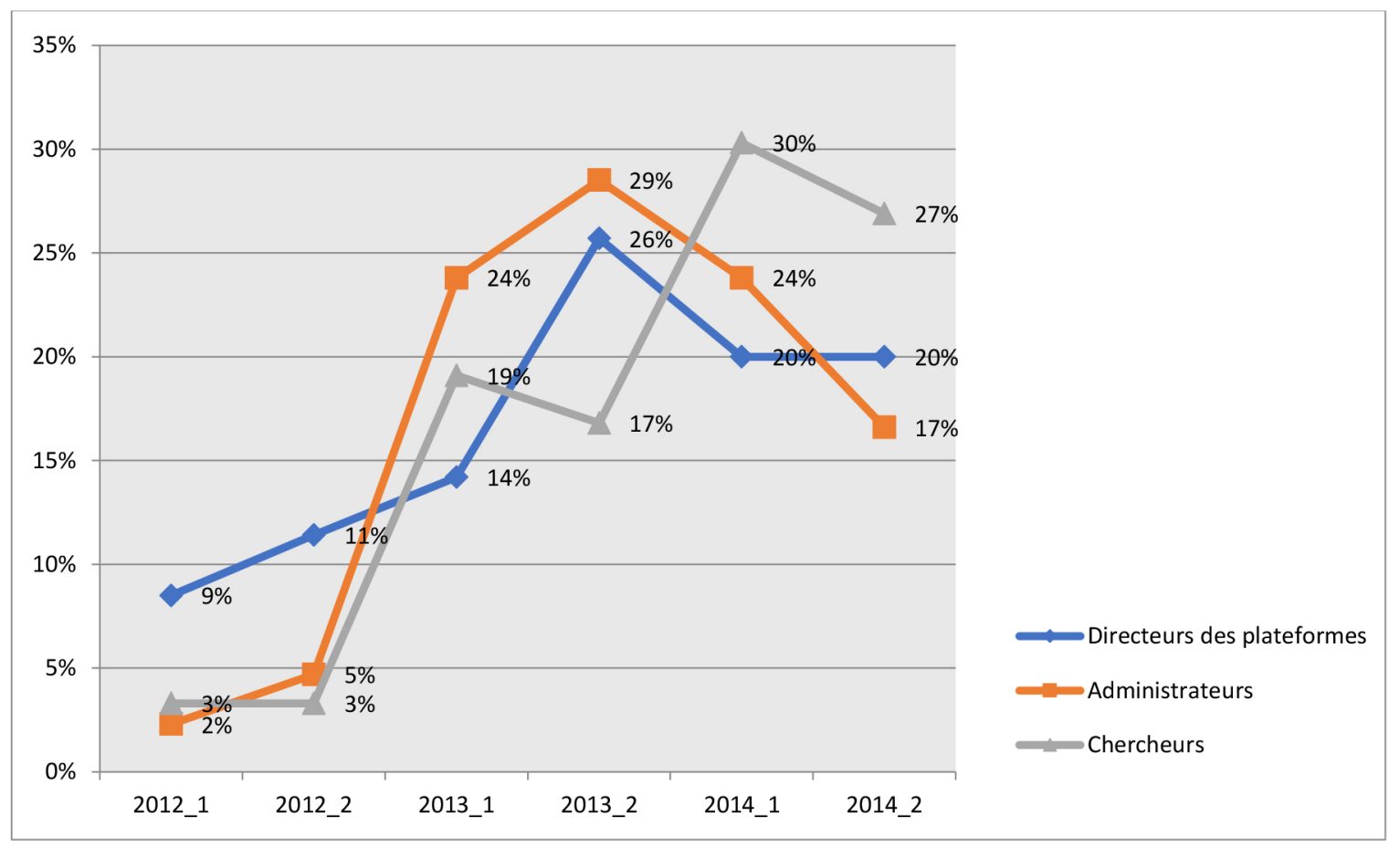

Dans la section suivante, nous reprenons les cinq codes principaux que nous avons identifiés dans le premier article (voir Collin et Saffari, 2015) et nous y répartissons les trois types d'intervenants (chercheurs, administrations universitaires, directions de plateformes de MOOC).

\subsection{Distribution des types d'intervenants selon les cinq codes principaux}

À la vue de la figure 2, il apparaît que la contextualisation historique et sociale caractérise pareillement les trois types d'intervenants par sa quasi-absence. Autrement dit, aucun de ces derniers n'est mobilisé plus que les autres de manière sensible dans les articles analysés.

Les autres codes principaux sont davantage exploités, quoique de manière inégale. Ainsi, la projection historique et sociale représente le troisième code principal le plus représenté. II est d'abord associé aux administrations universitaires (35\%), puis aux directions des plateformes de MOOC ( $27 \%)$, alors que les chercheurs y sont moitié moins représentés (16\%).

L'anticipation d'enjeux nouveaux suscités par les MOOC est le quatrième code principal le plus sollicité. Il est autant associé aux administrations universitaires (20\%) qu'aux chercheurs $(21 \%)$, alors que les directions des plateformes de MOOC y sont très peu représentées (3\%). 
La mise en doute critique forme le deuxième code principal le plus représenté. Les chercheurs y sont associés nettement plus souvent (41\%), suivis des directions de plateformes de MOOC (27 \%) et des administrations universitaires (18\%).

Finalement, les perspectives futures de développement des MOOC constituent le code principal le plus mobilisé dans les articles analysés. Les directeurs des plateformes de MOOC y sont nettement plus représentés (43\%), suivis des administrateurs (25\%) et des chercheurs ( $21 \%$ ) dans des proportions semblables.

Figure 2 - Distribution des types d'intervenants par codes principaux

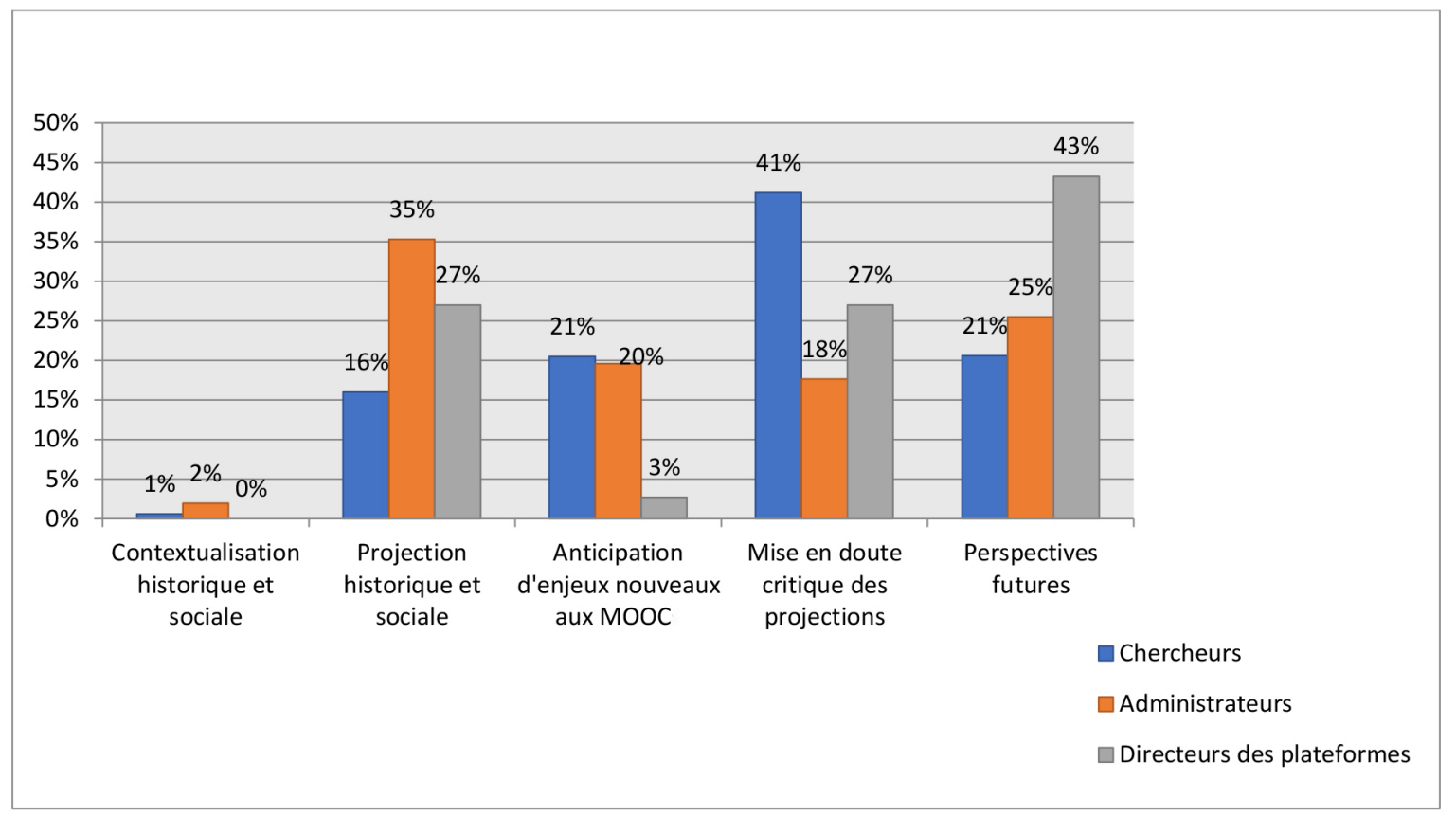

Nous pouvons maintenant approfondir ces premiers résultats en précisant pour chaque code principal quels sous-codes ont été privilégiés dans les articles analysés. Nous laissons toutefois de côté la contextualisation historique et sociale, faute d'être substantiellement abordée.

\subsection{Projection historique et sociale}

Les administrations universitaires sont massivement représentées dans les trois sous-codes les plus fréquents des projections historiques et sociales (voir figure 3). Le premier d'entre eux est également le plus consensuel entre les types d'intervenants ( $31 \%$ pour les chercheurs; $30 \%$ pour les directeurs des plateformes de MOOC; $29 \%$ pour les administrations universitaires) et concerne la démocratisation de l'accès à l'université.

Chercheurs: «Et ce n'est que le début! prédit Matthieu Cisel. [...] On va voir émerger des écoles qui n'ont pas un rond et qui pourtant auront tous les meilleurs professeurs du monde. En Europe, c'est en Grèce et en Espagne, 
touchés de plein fouet par la crise, que le nombre d'inscriptions aux Moocs augmente le plus.» (Le Parisien, 2013)

Le deuxième sous-code le plus fréquent est principalement associé aux administrations universitaires (35\%). Il s'agit de la visibilité académique et culturelle que les MOOC peuvent potentiellement procurer aux établissements d'enseignement supérieur.

Administration universitaire: "Le choix des MOOC n'est pas uniquement pédagogique: l'idée est aussi de soigner l'image de marque de Polytechnique et de répondre à la concurrence américaine, explique [Frank Pacard].» (Marmouyet, 2013)

Le troisième sous-code le plus fréquent est plus général que les deux premiers et projette les MOOC comme une révolution de l'enseignement supérieur. On le retrouve souvent en filigrane d'autres sous-codes dans le but de souligner l'ampleur des changements dont sont porteurs les MOOC.

Administration universitaire: "Stanford University President John Hennessey says "there's a tsunami coming"." (Auletta, 2012)

Pour leur part, les chercheurs sont représentés dans un plus grand nombre de souscodes ( 7 sur 9 ) que les autres intervenants (respectivement, 5 et 4 sous-codes pour les directions de plateformes de MOOC et les administrations universitaires).

Figure 3 - Distribution des types d'intervenants selon les projections historiques et sociales

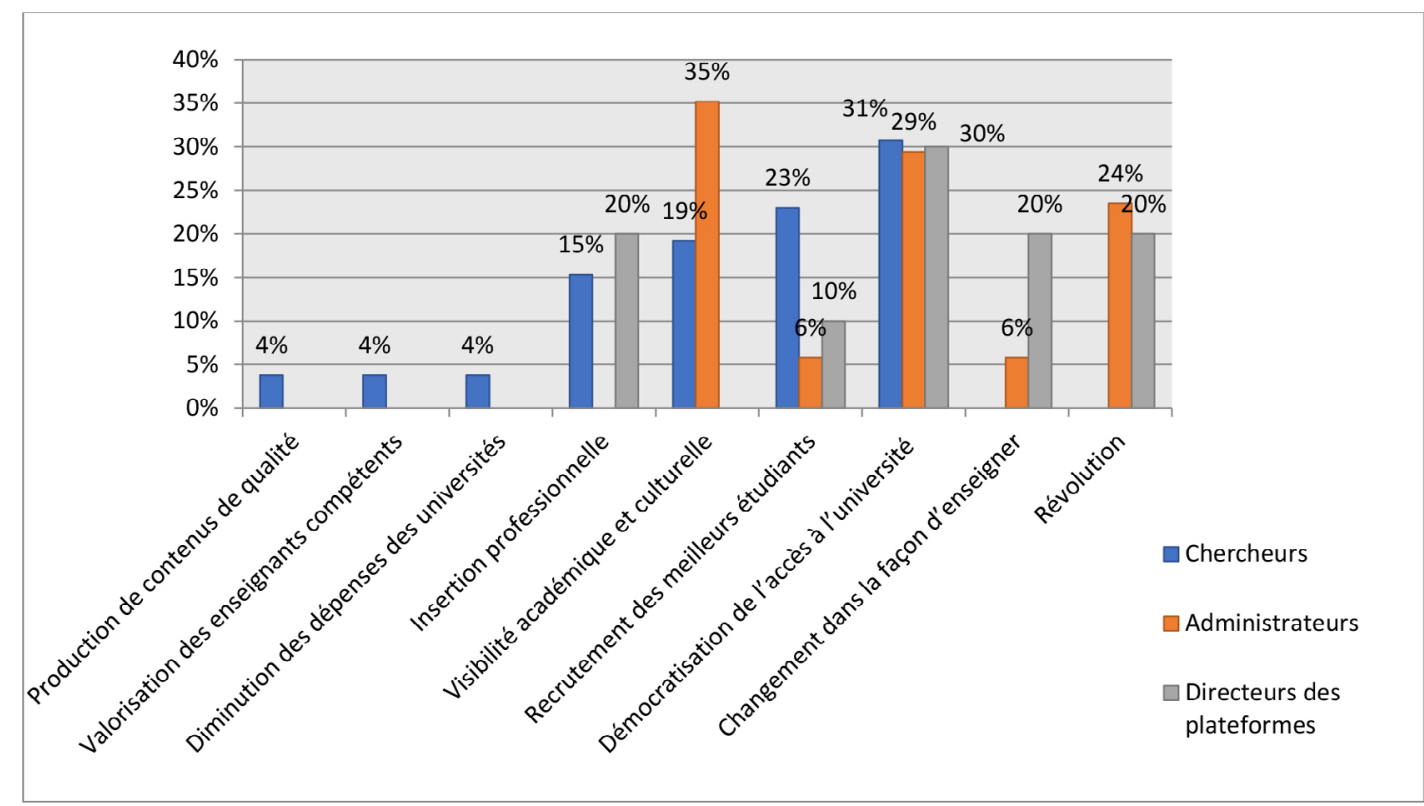

Les sous-codes secondaires font état d'une quasi-absence des administrations universitaires et se répartissent donc principalement entre les chercheurs et les directions des plateformes de MOOC. Ces deux types d'intervenants sont associés à des projections historiques et sociales telles que le recrutement des meilleurs étudiants, qui est souvent 
associé à la démocratisation d'accès à l'enseignement supérieur, ainsi que la facilitation de l'insertion professionnelle et l'impulsion de changements pédagogiques.

\subsection{Anticipation d'enjeux nouveaux suscités par les MOOC}

La figure 4 permet de constater que les types d'intervenants ne sont pas représentés pareillement dans l'anticipation d'enjeux nouveaux suscités par les MOOC. En effet, les administrations universitaires sont principalement mobilisées (50 \%) en lien avec un souscode, celui de l'incertaine acceptation et valorisation des certificats délivrés par les MOOC:

Administration universitaire: "But when someone receives a degree from Duke or Stanford or MIT, people know that means something. Those things are less certain or less sure when you're dealing with a MOOC." (Beth Marklein, 2012)

\section{Figure 4 - Distribution des types d'intervenants selon l'anticipation d'enjeux nouveaux suscités par les MOOC}

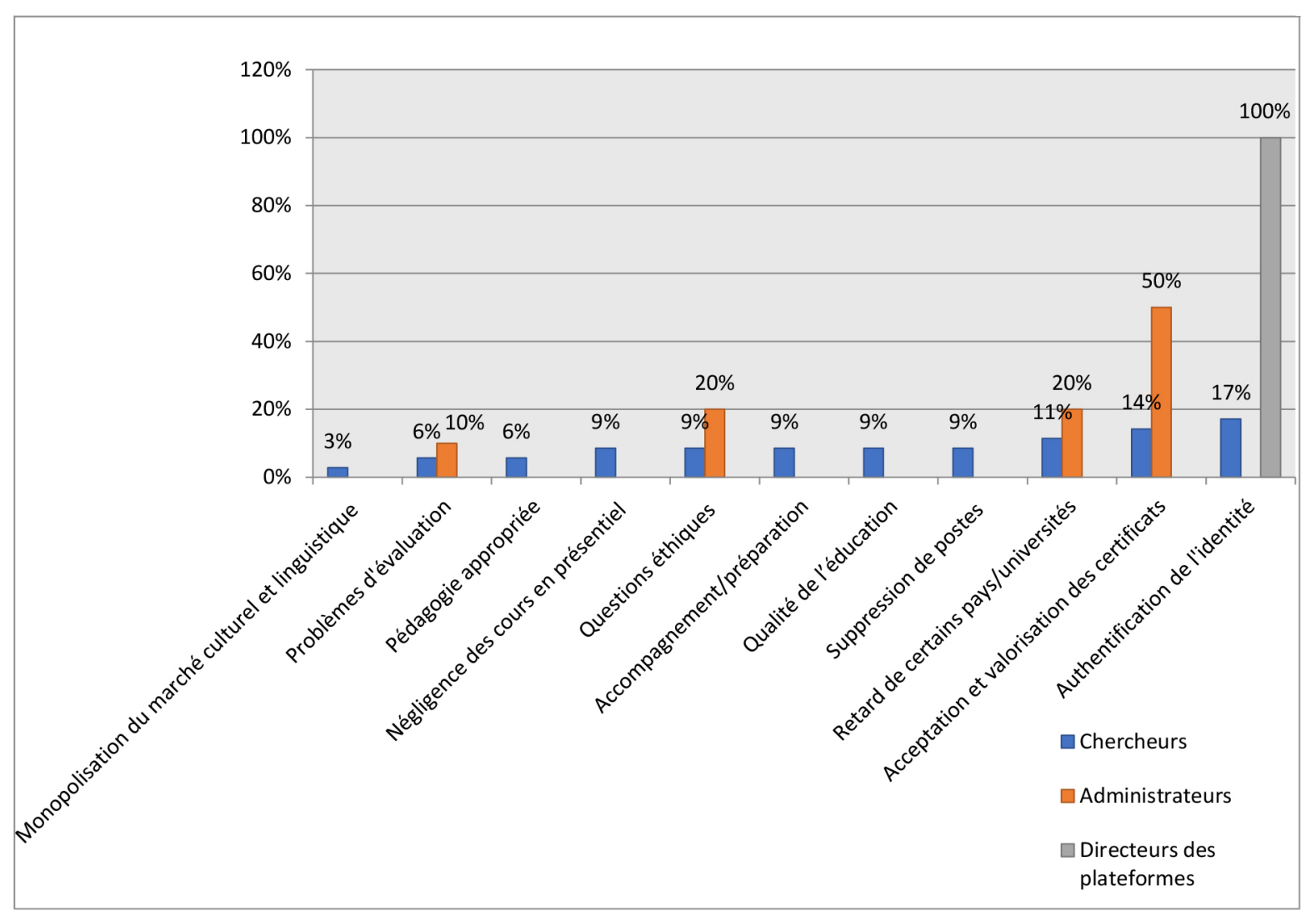

Les trois autres sous-codes associés aux administrations universitaires sont secondaires et touchent autant à des aspects institutionnels (p. ex., retard de certaines universités ou de pays dans le développement de MOOC; questions éthiques liées à la protection des données des participants par les plateformes de MOOC) qu'à des aspects pédagogiques (p. ex., problèmes d'évaluation des étudiants participants aux MOOC). 
De façon nette, les chercheurs sont représentés dans tous les sous-codes identifiés, et ce, de façon beaucoup plus égale, ce qui témoigne a priori d'une vision plus large des enjeux nouveaux suscités par les MOOC. Les trois principaux sous-codes auxquels ils sont associés sont l'authentification de l'identité des étudiants (17 \%), notamment en lien avec l'évaluation et l'attribution de certificat de réussite, l'acceptation et la valorisation des certificats (14\%), et le retard de certaines universités ou de pays dans le développement de MOOC (11\%).

Finalement, les directions de plateformes de MOOC sont exclusivement mobilisés en lien avec le sous-code de l'authenticité de l'identité des étudiants (100\%), notamment en lien avec l'évaluation et l'attribution de certificat de réussite.

\subsection{Mise en doute critique}

Concernant la mise en doute critique, deux sous-codes sont communs aux trois types d'intervenants (voir figure 5), dont, en premier lieu, l'absence d'un modèle d'affaires viable pour financer les MOOC.

Chercheur: "There still is not a clear business model to why I should do this," Mr. Seaman said." (Drake, 2014)

Le fort taux d'abandon des étudiants inscrits aux MOOC est le second sous-code qui mobilise les trois types de participants.

Directions: «Très peu d'élus finissent leurs cours. M. Levin l'avoue tout net. $10 \%$ seulement décrocheront leur certificat.» (Lambert-Chan, 2013)

Les sous-codes restants sont spécifiques à certains types de participants uniquement et sont moins fréquemment rapportés. Ainsi, la gratuité toute relative des MOOC est uniquement (et fortement) associée aux administrations universitaires (22\%):

Administration: "Les MOOC font un carton. Mais, ces cours en ligne ouverts à tous ne sont pas gratuits, prévient Ludovic Legris, senior manager éducation chez Kurt Salmon.» (Salmon, 2014)

Les chercheurs, pour leur part, sont les seuls à être mobilisés pour les cinq sous-codes, en particulier la mise en doute critique des MOOC comme un moyen de démocratiser l'accès à l'enseignement supérieur (17 \%).

Chercheurs: «Fiona M. Hollands and Devayani Tirthali of Columbia University Teachers College [argues] that most MOOC participants are already well-educated and employed, and only a small fraction of them fully engages with the courses. Overall, the evidence suggests that MOOCs are currently falling far short of "democratizing" education and may, for now, be doing more to increase gaps in access to education than to diminish them." (North, 2014) 
Les sous-codes restants sont moins fréquemment rapportés (11\% ou moins de mentions) et ne sont jamais associés aux trois types d'intervenants simultanément, ce qui explique pourquoi nous ne les détaillons pas davantage. En écho aux résultats précédents, une tendance notable a trait au fait que les chercheurs sont mobilisés pour un nombre de sous-codes beaucoup plus élevé (11 sur 12) que les deux autres types d'intervenants.

Figure 5 - Distribution des types d'intervenants selon les mises en doute critiques des MOOC

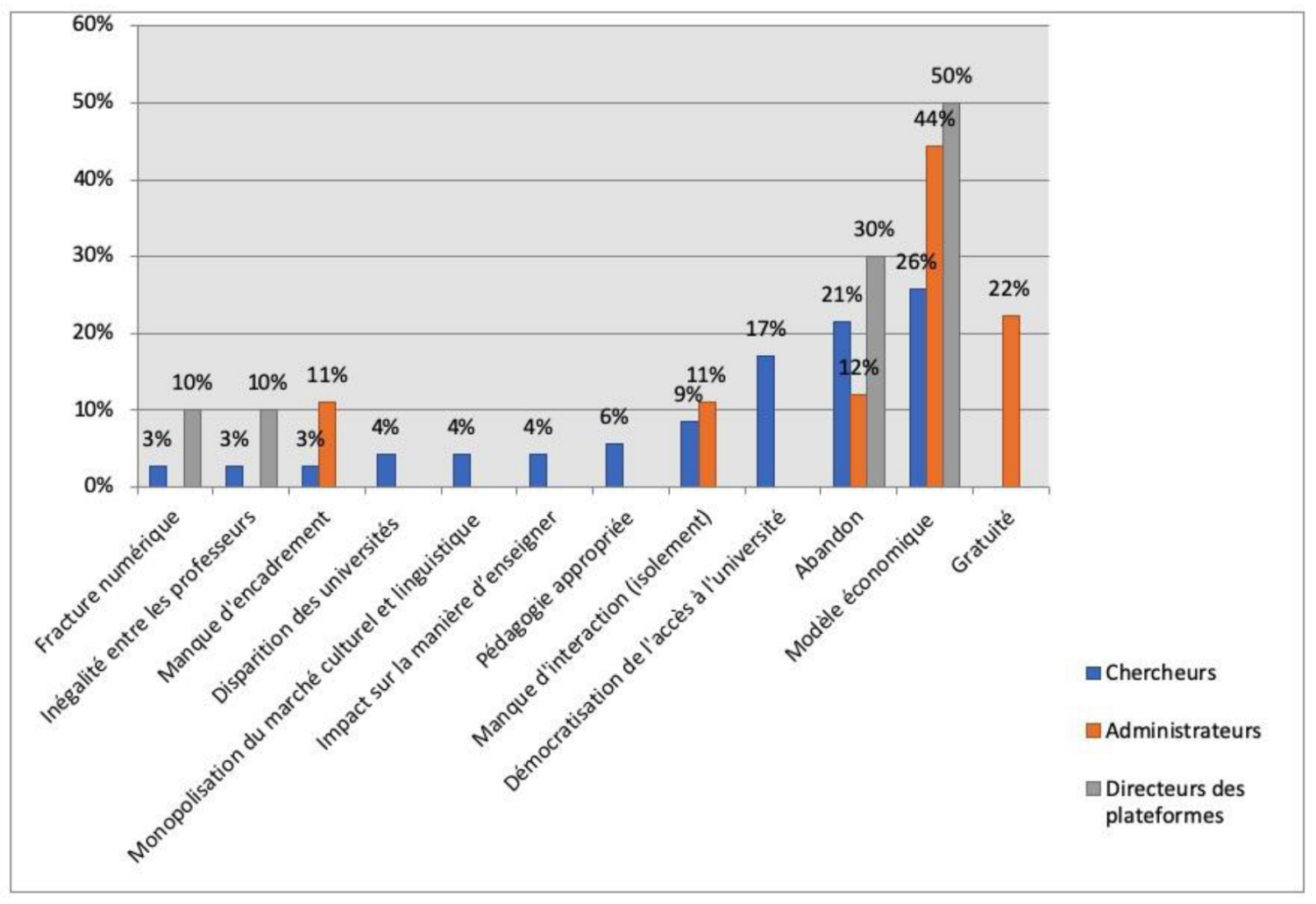

\subsection{Perspectives futures}

À l'instar des mises en doute critiques, les sous-codes sont associés différemment à chaque type d'intervenants (voir figure 6). Ainsi, les chercheurs sont mobilisés plus fréquemment en lien avec l'autoformation comme un avenir possible des MOOC (54 \%) que les autres types d'intervenants (31\% pour les administrations universitaires et les directions de plateformes de $\mathrm{MOOC}$ ), notamment en réponse au fort taux d'abandon des MOOC par les étudiants.

Chercheurs: “Quant aux apprenants, ce n'est pas parce qu'ils ont abandonné qu'ils n'y ont pas trouvé leur compte. Certains ne font que piocher de façon ponctuelle les informations qui les intéressent." (Grenouillet, 2014) 
Les administrations universitaires sont associées plus fréquemment que les autres (46\% contre $29 \%$ pour les chercheurs et $25 \%$ pour les directions de plateformes de MOOC) aux MOOC comme des outils complémentaires aux cours dispensés en présentiel.

Administrations universitaires: "Mais, pour lui, tout cela ne préfigure en rien un basculement de l'enseignement traditionnel vers les Mooc. Le travail de formation se fait d'abord en présentiel. Au mieux pourraiton imaginer des modules complémentaires en ligne.” (Soulé, 2014)

Elles sont également plus nombreuses que les autres intervenants ( $23 \%$ contre $14 \%$ pour les chercheurs et $13 \%$ pour les directions de plateformes MOOC) à être associées à l'avenir des MOOC en adaptant leur format actuel pour qu'il soit plus adéquat aux études universitaires.

Finalement, les directions de plateformes de MOOC sont quasiment les seules à être mobilisées au sujet de l'avenir des MOOC en lien avec la formation professionnelle:

Administration des MOOC: "Yet when Udacity founder Sebastian Thrun revealed in a Fast Company interview that his company is abandoning higher education and moving to corporate training, he managed to recapture our attention in one fell swoop." (Kelly, 2014)

Ceci s'explique sans doute par le fait que la formation professionnelle ne concerne pas le contexte universitaire, de sorte qu'elle échappe aux administrations universitaires et aux chercheurs qui y œuvrent.

Figure 6 - Distribution des types d'intervenants selon les perspectives futures des MOOC

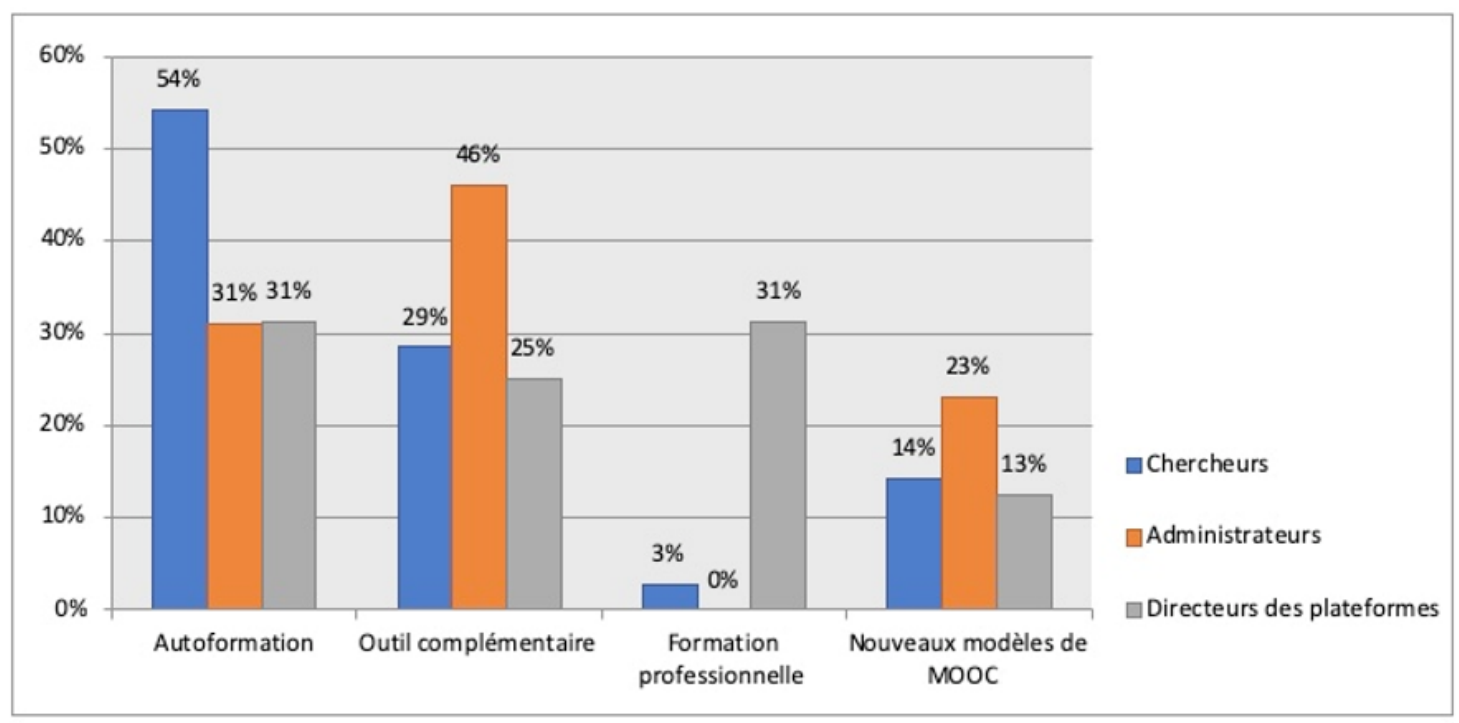

En somme, il apparaît que les trois types d'intervenants étudiés sont mobilisés différemment, à la fois sur le plan des codes principaux et des sous-codes. La discussion nous permet de préciser ces positionnements. 


\section{Discussion et conclusion}

Pour rappel, l'objectif de ce texte était de mieux comprendre comment le traitement médiatique des MOOC positionne les chercheurs, les administrations universitaires et les directions de plateformes de MOOC au sein de l'imaginaire qu'ils véhiculent. L'analyse du corpus d'article de ces trois types d'intervenants révèle des positionnements complémentaires, liés à des rôles différents.

Ainsi, en faisant une relecture de la figure 2 , on constate que les administrateurs universitaires sont mobilisés en première position des projections historiques et sociales sur le potentiel des MOOC. Ils apparaissent aussi, à parts égales avec les chercheurs, dans l'anticipation d'enjeux nouveaux suscités par les MOOC. Ils semblent donc essentiellement positionnés pour alimenter l'attrait initial pour les MOOC et l'identification de problèmes pédagogiques et institutionnels lorsqu'ils surviennent. Il est possible d'avancer que leur positionnement dans le traitement médiatique des MOOC a pour but de «mettre en valeur la capacité d'adaptation et de modernisation [de leurs] établissements" (Albero, 2011, p. 15). Preuve à l'appui: la visibilité académique et culturelle des universités constitue un code particulièrement associé aux administrations universitaires dans leurs projections initiales sur les MOOC (voir figure 3).

Les chercheurs sont moins associés que les administrations universitaires et les directions de plateformes de MOOC aux projections historiques et sociales, ainsi qu'aux perspectives d'avenir pour les MOOC (voir figure 2). À l'inverse, ils sont particulièrement mobilisés en lien avec l'anticipation d'enjeux nouveaux suscités par les MOOC et la mise en doute critique des projections historiques et sociales initialement attribuées aux MOOC. Dans ces deux codes principaux, ils sont représentés dans un nombre bien plus grand de sous-codes que les deux autres intervenants, ce qui semble faire état d'une vision plus large des tenants et aboutissants des MOOC. Il est possible d'avancer que leur positionnement dans le traitement médiatique des MOOC tient lieu de caution scientifique, laquelle est d'autant plus nécessaire qu'elle porte sur une innovation récente, donc encore mal connue du grand public. Il ressort de notre analyse que les chercheurs se voient attribuer un rôle positif dans le traitement médiatique des MOOC dans la mesure où ils contribuent à contenir et à orienter les projections des deux autres types d'intervenants.

Les directions des plateformes de de MOOC sont positionnées au premier rang des perspectives futures des $\mathrm{MOOC}$ (voir figure 2). On les retrouve également au deuxième rang des projections historiques et sociales et des mises en doute critiques. Plus précisément, ces dernières portent soit sur l'absence d'un modèle d'affaires viable pour financer les MOOC, soit sur les freins à l'expansion du bassin d'usagers des MOOC (fort abandon 
des étudiants; inégalités entre les professeurs; fractures numériques entre les pays) (voir figure 5). Ainsi, ce type d'intervenant semble à la fois mobilisés pour alimenter le potentiel initial des MOOC et pour identifier certaines limites critiques qui pourraient entraver leur diffusion. Fait intéressant: parmi les trois intervenants, les directions de plateformes de MOOC sont les seules à ne pas être attachées au monde universitaire. Ceci explique sans doute pourquoi elles sont fortement associées à l'idée que la formation professionnelle constitue une voie d'avenir prometteuse (voir figure 6): cette dernière pourrait s'avérer davantage lucrative que les universités tout en ouvrant sur de nouveaux bassins d'usagers potentiels, résolvant ainsi les mises en doute critiques identifiées préalablement. En somme, le positionnement des directions de plateformes de MOOC dans le traitement médiatique des MOOC semble essentiellement consister à assurer la survie de l'innovation, de diverses façons: en témoignant initialement de son potentiel; en identifiant les limites critiques qui pourraient entraver sa diffusion; finalement, en s'en servant pour ouvrir sur des perspectives plus prometteuses, quitte à passer du marché universitaire au marché entrepreneurial.

Ainsi, l'analyse des propos de trois types d'intervenants dans le traitement médiatique des MOOC révèle des positionnements complémentaires au sein de l'imaginaire que cette innovation numérique a suscité en éducation: opération stratégique pour les administrations universitaires; caution scientifique pour les chercheurs; survie de l'innovation pour les directions des plateformes de MOOC.

Pour approfondir l'étude de l'imaginaire des MOOC en tant qu'innovation numérique en éducation, il serait intéressant, en premier lieu, de reproduire l'analyse sur la période allant de 2015 à nos jours. En effet, en reprenant les trois étapes de l'appropriation sociale d'une innovation technique de Musso et al. (2007), à savoir, les discours prophétiques sur l'innovation technique (étape 1); la diffusion sociale de l'innovation technique et désillusion (étape 2); et la réappropriation culturelle de l'innovation technique (étape 3), il est possible de penser que la "période médiatique" couverte dans le présent article correspond surtout aux phases 1 et 2 et au début de la phase 3 (Collin et Saffari, 2015). Il serait intéressant de reproduire l'analyse depuis 2015 pour documenter la manière dont l'imaginaire véhiculé par les médias a évolué, notamment en ce qui a trait à la mise en scène des acteurs concernés, alors que les MOOC se sont stabilisés dans le paysage universitaire.

En deuxième lieu, il serait intéressant de confronter le positionnement médiatique des trois types d'intervenants à leur autopositionnement, au moyen d'entrevues auprès des chercheurs, des administrations universitaires et des directions de plateformes de MOOC. Cette piste de recherche future permettrait d'interroger la marge de décalage de l'imaginaire médiatique par rapport à la réalité perçue des acteurs concernés. 
Finalement, et bien que nous n'ayons pas d'explications convaincantes à ce sujet, le traitement médiatique a principalement mis en scène trois types d'intervenants. En revanche, d'autres intervenants en lien avec les MOOC, notamment ceux mentionnés par Roland et Uyttebrouck (2015), ont été sollicités de façon moindre dans les articles analysés. II s'agit notamment des enseignants universitaires, des étudiants, des services de soutien technopédagogique et des instances politiques. Il serait intéressant de chercher à savoir si ces acteurs peu mobilisés dans les articles journalistiques généralistes ont été effectivement exclus du traitement médiatique des MOOC ou s'ils n'y participent pas sous d'autres formes médiatiques (p. ex., blogs, réseaux sociaux, etc.).

\section{Références}

Albero, B. (2011). Le couplage entre pédagogie et technologies à l'université: cultures d'action et paradigmes de recherche. Revue internationale des technologies en pédagogie universitaire, 8(1-2), 11-21. Repéré à http://www.ritpu.org/IMG/pdf/RITPU_v08_n01-02_11.pdf.

Auletta, K. (2012). Get Rich U. The New Yorker, 30 avril. Repéré à http://www.newyorker.com/ magazine/2012/04/30/get-rich-u.

Beth Marklein, M. (2012). College may never be the same. Usa Today, 9 décembre. Repéré à https://usatoday30.usatoday.com/news/nation/story/2012/09/12/college-may-never-bethe-same/57752972/1.

Chanial, P. (1994). Entretien avec Georges Balandier. Quaderni, 23, "Science(s) de la communication".

Collin, S. et Saffari, H. (2015). Le MOOC et le "hype»: analyse critique des discours médiatiques sur les MOOC. Revue internationale des technologies en pédagogie universitaire/International Journal of Technologies in Higher Education, 12(1-2), 124-137.

Drake, M. (2014). Old School Rules! Wisdom of massive open online courses now in doubt. The Washington Times, 9 février. Repéré à http://m.washingtontimes.com/news/2014/ feb/9/big-plan-on-campus-is-dropping-out/.

Forestier, Y. (2014). La primauté d'une querelle providentielle. Les questions pédagogiques dans la presse française (années 1960-années 2000). Revue internationale d'éducation de Sèvres, 66(2), 43-52. Repéré à https://journals.openedition.org/ries/3993.

Friesen, N. (2008). Critical theory: Ideology critique and the myths of e-learning. Ubiquity, 9(22). Repéré à https://doi.org/10.1145/1403922.1386860.

Garçon, A. F. (2006). Les techniques et l'imaginaire. Hypothèses, 9(1), 221-228. Repéré à https:// www.cairn.info/revue-hypotheses-2006-1-page-221.htm.

Gérard, É., et Proteau, L. (2002). Les «jeunes» en mots. Discours journalistiques et enjeux politiques en Afrique. Cahiers de la recherche sur l'éducation et les savoirs, 1, 7-15. Repéré à https://journals.openedition.org/cres/1651.

Grenouillet, E. (2014). Un MOOC ne remplacera jamais un cours traditionnel. Le Figaro, $1^{\mathrm{er}}$ avril. Repéré à http://www.lefigaro.fr/éducation/enligne/moocneremplacerajamais.

Inaudi, A. (2017). École et numérique: une histoire pour préparer demain. Hermes, La Revue, 78(2), 72-79. Repéré à https://www.cairn.info/revue-hermes-la-revue-2017-2-page-72.htm.

Karsenti, T. (2013). MOOC: révolution ou simple effet de mode? Revue internationale des technologies en pédagogie universitaire, 10(2), 6-22. Repéré à http://ritpu.org:81/img/pdf/ RITPU_v10_n02_06.pdf. 
Karsenti, T., Komis, V., Depover, C. et Collin, S. (2011). Les TIC comme outils de recherche en sciences de l'éducation. Dans T. Karsenti et L. Savoie-Zajc (dir.), La recherche en éducation: étapes et approches (p. 168-192). Saint-Laurent, QC: ERPI.

Kelly, R. (2014). Beyond the MOOC hype. Campus technology, $1^{\text {er }}$ août. Repéré à https://campustechnology.com/articles/2014/01/08/beyond-the-mooc-hype. aspx?admgarea=News.

Lambert-Chan, M. (2013). Université - À revoir, les MOOC! Le Devoir, 14 décembre. Repéré à http://www.ledevoir.com/societe/education/394927/a-revoir-les-mooc.

Le Blanc, B. (2017). Entre lendemains meilleurs et apocalypse imminente: ce que disent les médias du numérique à l'école. Hermes, La Revue, 78(2), 62-64. Repéré à https://www.cairn. info/revue-hermes-la-revue-2017-2-page-62.htm.

Le Parisien (2013). On peut tous faire Harvard. 25 août. Repéré à http://www.leparisien.fr/espacepremium/air-du-temps/on-peut-tous-faire-harvard-25-08-2013-3077881.php.

Marmouyet, F. (2013). Vers des cours en ligne pour tous. La Croix, 2 octobre. Repéré à http:// www.la-croix.com/Monde/Vers-des-cours-en-ligne-pour-tous-2013-10-02-1031406.

Millerand, F. (1999). Usages des NTIC: les approches de l'innovation, de la diffusion et de l'appropriation ( $2^{\mathrm{e}}$ partie). Repéré à http://com2710.dedalon.net/S_03_files/ MILLERAND_1999_Usages_des_NTIC.pdf.

Musso, P. (2009). Usages et imaginaires des TIC. Repéré à https://hal-imt.archives-ouvertes.fr/ hal-00479606/document.

Musso, P., Ponthou, L. et Seulliet, É. (2007). Fabriquer le futur 2. L'imaginaire au service de l'innovation. Paris: Village mondial.

North, A. (2014). Does online education widen offline gaps? The New York Times, 23 septembre. Repéré à https://op-talk.blogs.nytimes.com/2014/09/23/does-online-education-widenoffline-gaps/.

Oliveri, N. et Rasse, P. (2017). Les Mooc et leurs dérivés, ou l'imaginaire des technologies pédagogiques. Hermès, La Revue, 78(2), 110-117. Repéré à https://www.cairn.info/revuehermes-la-revue-2017-2-page-110.htm.

Roland, N. et Uyttebrouck, E. (2015). Critique de l'innovation technopédagogique dans l'enseignement supérieur. Dans Actes du VIII colloque Questions de pédagogie dans l'enseignement supérieur. Innover: comment et pourquoi? du 17 au 19 juin 2015 à Brest, France (p. 51-60). Repéré à http://www.colloque-pedagogie.org.

Salmon, K. (2014). MOOC: effet de mode ou tendance durable? L'Express, 13 mai. Repéré à http:// www.lexpress.fr/actualite/mooc-un-effet-de-mode-ou-une-tendance-durable_1543092.html.

Scardigli, V. (1992). Les sens de la technique. Paris: Presses Universitaires de France.

Soulé, V. (2014). Internautes de tous les pays, instruisez-vous. Liberation, 20 octobre. Repéré à http:// www.liberation.fr/futurs/2013/10/20/internautes-de-tous-les-pays-instruisez-vous_940986.

Van der Maren, J.-M. (1996). Méthodes de recherche pour l'éducation. (2 édition). Montréal, QC: Presses de l'Université de Montréal.

Wilby, P. (2014). Vers de nouveaux récits médiatiques sur l'école au Royaume-Uni? Revue internationale d'éducation de Sèvres, 66(2), 31-42 Repéré à https://journals.openedition.org/ ries/3990. 